\title{
Anatomical and Functional Recovery of Neurotized Remnant Rectus Abdominis Muscle in Muscle-Sparing Pedicled Transverse Rectus Abdominis Musculocutaneous Flap
}

\author{
Woonhyeok Jeong ${ }^{1}$, Daegu Son ${ }^{1}$, Hyeonjung Yeo ${ }^{1}$, Hoijoon Jeong ${ }^{1}$, Junhyung Kim ${ }^{1}$, \\ Kihwan $\mathrm{Han}^{1}$, Soyoung Lee ${ }^{2}$ \\ Departments of ${ }^{1}$ Plastic and Reconstructive Surgery and ${ }^{2}$ Rehabilitation Medicine, Keimyung University Dongsan Medical Center, Keimyung \\ University School of Medicine, Daegu, Korea
}

Background Pedicled transverse rectus abdominis musculocutaneous flaps typically sacrifice the entire muscle. In our experience, the lateral strip of the rectus abdominis muscle can be spared in an attempt to maintain function and reduce morbidity. When the intercostal nerves are injured, muscle atrophy appears with time. The severed intercostal nerve was reinserted into the remnant lateral strip of the rectus abdominis muscle to reduce muscle atrophy.

Methods The authors retrospectively reviewed 9 neurotized cases and 10 non-neurotized cases. Abdominal computed tomography was performed to determine the area of the rectus muscles. Electromyography (EMG) was performed to check contractile function of the remnant muscle. A single investigator measured the mean areas of randomly selected locations (second lumbar spine) using ImageJ software in a series of 10 cross-sectional slices. We compared the Hounsfield unit (HU) pre- and postoperatively to evaluate regeneration quality.

Results In the neurotization group, 7 of 9 cases maintained the mass of remnant muscle. However, in the non-neurotization group, 8 of 10 lost their mass. The number of totally atrophied muscles in each of the two groups was significantly different $(P=0.027)$. All of the remnant muscles showed contractile function on EMG. The 9 remaining remnant rectus abdominis muscles showed declined the HU value after surgery but also within a normal range of muscle.

Conclusions Neurotization was found to be effective in maintaining the mass of remnant muscle. Neurotized remnant muscle had contractile function on EMG and no fatty degeneration by $\mathrm{HU}$ value.

Keywords Rectus abdominis / Nerve transfer / Electromyography / Multidetector computed tomography
Correspondence: Daegu Son

Department of Plastic and Reconstructive Surgery, Keimyung University Dongsan Medical Center, Keimyung University School of Medicine, 56 Dalseong-ro, Jung-gu, Daegu 700-712, Korea

Tel: $+82-53-250-7636$

Fax: +82-53-255-0632

E-mail: handson@dsmc.or.kr

This article was presented at the Research and Reconstructive Forum on May 12-13, 2011 in Daejeon, Korea.

The authors thank Dr. Yang for the medical illustrations

No potential conflict of interest relevant to this article was reported.

Received: 7 Mar 2013 • Revised: 20 May 2013 • Accepted: 13 Jun 2013

pISSN: 2234-6163 • elSSN: 2234-6171 • http://dx.doi.org/10.5999/aps.2013.40.4.359 • Arch Plast Surg 2013;40:359-366

Copyright ( $) 2013$ The Korean Society of Plastic and Reconstructive Surgeons

This is an Open Access article distributed under the terms of the Creative Commons Attribution Non-Commercial License (http://creativecommons.org/

licenses/by-nc/3.0/) which permits unrestricted non-commercial use, distribution, and reproduction in any medium, provided the original work is properly cited.

www.e-aps.org 


\section{INTRODUCTION}

Since the first description of a pedicled transverse rectus abdominis musculocutaneous (TRAM) flap by Hartrampf et al. [1] in 1982, it has been the preferred method for breast reconstruction after mastectomy. Because the rectus abdominis muscle is sacrificed in a pedicled TRAM, it can result in donor site morbidity, such as weakness of the abdominal wall and hernia [2]. Musclepreserving TRAM [3] and free TRAM were developed in order to overcome donor site morbidity. Although free TRAM has been proposed in order to avoid many of the shortcomings of pedicled TRAM, such as donor site morbidity and reduced flap vascularity, it demands advanced microsurgical skill and prolonged operative time. Controversy exists regarding the difference in complications between free TRAM and pedicled TRAM [4-7]. Mizgala et al. [5] reported significant abdominal laxity in patients undergoing pedicled TRAM. On the other hand, Suominen et al. [7] reported that there were no significant differences in abdominal laxity between free TRAM and pedicled TRAM. Alderman et al. [8] also reported no significant difference in trunk flexion on objective measures of physical function. Therefore, free TRAM is not always a superior method to pedicled TRAM.

Hartrampf [3] introduced a muscle-sparing technique for pedicled TRAM, leaving the lateral third of the muscle. The intercostal nerves enter the posterior surface of the rectus abdominis muscle in the lateral third, through which the lateral row of the superior and inferior epigastric arteries pass [9]. Therefore, intercostal nerves are vulnerable in the medial two thirds of the muscle harvest. Lejour and Dome [10] reported no significant difference in abdominal function between musclesparing pedicled TRAM and conventional pedicled TRAM due to fibrosis of the remnant muscle. If the remnant rectus muscle is preserved, rather than atrophied, it can result in avoidance of abdominal wall dysfunction. The muscle-sparing technique also ensures less epigastric bulging due to the lower quantity of muscle bulk that needs to fold at the pivot point of the flap [11].

To prevent atrophy, the author performed neurotization by the simple transplanting of the severed intercostal nerve to the spared lateral third of the rectus abdominis muscle. The degree of atrophy of the remnant rectus muscle was determined by measuring the cross-sectional area of the preoperative and postoperative rectus abdominis muscles using abdominal computed tomography (CT). Electromyography (EMG) was performed to determine the contractile function of the remnant muscle.

\section{METHODS}

\section{Subjects}

Nineteen patients who underwent muscle-sparing pedicled TRAM flap done by a single senior surgeon from 2006 to 2010 were reviewed retrospectively. Nine patients underwent neurotized muscle-sparing pedicled TRAM flap and 10 patients underwent non-neurotized muscle-sparing pedicled TRAM flap. Neurotization was defined as only reinsertion of the severed nerve to the remnant rectus muscle, excluding the case of a naturally preserved nerve. The study was approved by the Institutional Ethics Committee. The patients gave consent to participate in this study after receiving sufficient explanation of the purpose, methods, and potential complications. There was no postoperative treatment for preventing the atrophy of the rectus muscle.

\section{Surgical technique}

The abdominal flap was dissected beyond the costal margin, approximately $5 \mathrm{~cm}$. The TRAM flap was dissected medially until the lateral perforators of the deep inferior epigastric artery were encountered. Then, the anterior rectus sheath was incised longitudinally at just the lateral side of the perforators. The rectus abdominis muscle was separated from the rectus sheath. The intercostal nerve and vessel appeared on the posterior surface of the rectus abdominis muscle (Fig. 1A). After the rectus abdominis muscle was totally separated from the rectus sheath with preservation of the intercostal nerve and vessel, the rectus abdominis muscle was split longitudinally on the lateral side to the lateral row of the deep inferior epigastric and superior epigastric artery. The lateral rectus abdominis muscle was left on the abdominal wall, and the other medial rectus muscle including the pedicle vessels was harvested with a TRAM flap. The width of the remnant muscle on the abdominal wall was about 2 to $3 \mathrm{~cm}$, and it was similar to MS-1 of Nahabedian's classification. Therefore, the intercostal nerve innervating the medial side to the superior and inferior epigastric arteries could not be preserved (Fig. 1B). We attempted to preserve the intercostal nerve, especially the large one innervating at the arcuate line, but the nerves innervating close to the pedicle could not be preserved in order to guarantee the safety of the vascularity. The nerves were severed to avoid pedicle injury and transposed to the remnant rectus abdominis muscle by neurotization. Neurotization is reinsertion of the severed nerve to remnant muscle. Before the intercostal nerve was severed, the accompanying intercostal vessels were divided meticulously and coagulated using bipolar electrocautery. Then, the intercostal nerve was severed with as clean a margin as possible using Metzenbaum scissors. The intercostal nerve was 


\section{Fig. 1. Schema of surgical anatomy and neurotization}

(A) Innervation of the rectus abdominis muscle by intercostal nerves. Intercostal nerves innervating the lateral side of the pedicle (red arrow) can be preserved during harvest of the rectus muscle. The two middle nerves innervating medial to the pedicle (red arrow head) may be severed during muscle harvesting. (B) Trans-sectional view: The intercostal nerve could be severed during muscle harvesting. (C) The severed intercostal nerve is re-innervated to the remaining muscle using 9-0 non-absorbable suture.
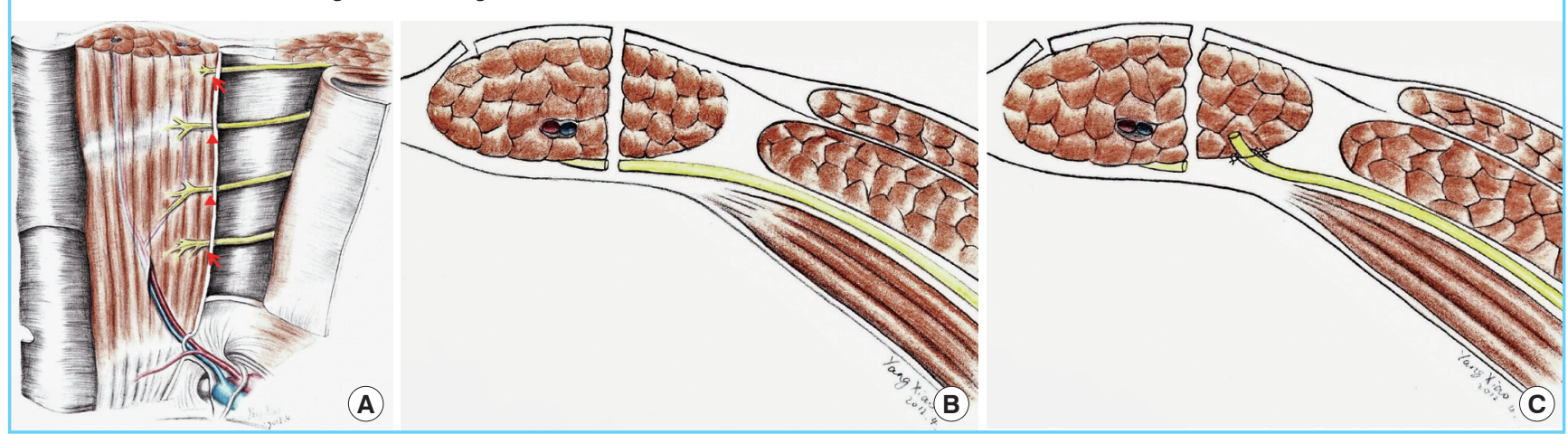

\section{Fig. 2. Preservation and neurotization of the intercostal nerve}

(A) All intercostal nerves innervating the rectus muscle can be preserved during muscle harvesting. However, in the author's experience, such cases were rare. (B) One intercostal nerve (red arrow) was sacrificed due to its location, which was close to the pedicle. (C) All of the intercostal nerves were severed during muscle harvesting. The severed nerves (blue arrow) were re-innervated to the remaining lateral strip of the rectus muscle by means of neurotization.
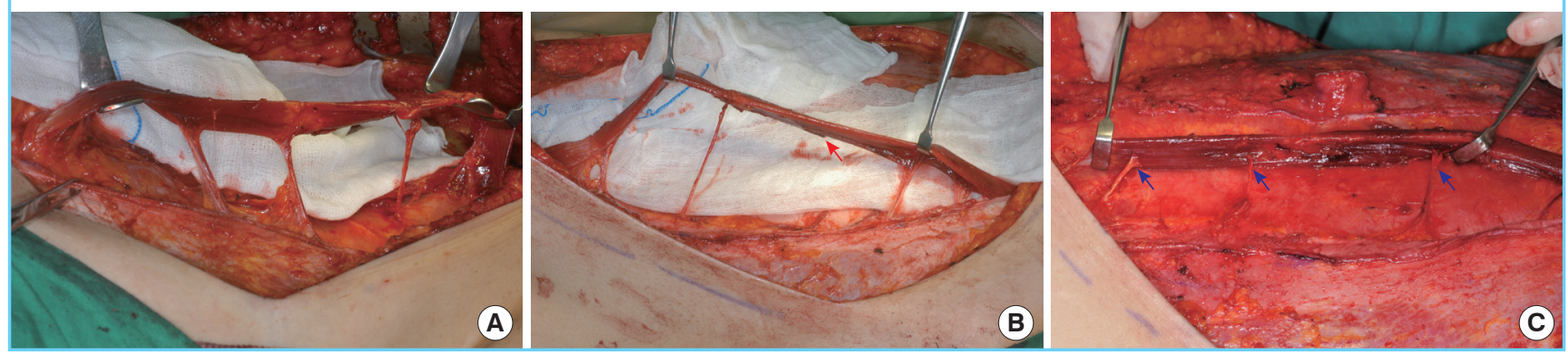

placed at the remnant rectus abdominis muscle tension-free and inserted between the muscle fibers, and the epineurium was sutured to the epimysium of the muscle with 9-0 nylon (Fig. 1C). The neurotization was performed with at least two intercostal nerves, including the large one at the arcuate line (Fig. 2). A previous investigator reported preserving the medial strip of the rectus abdominis muscle in order to aid the final result of the donor site; however, the author concluded that this was an unnecessary procedure because the denervated muscle eventually atrophied [6]. The elevated flap was inset to the mastectomy defect and then the anterior rectus fascia and remaining muscle was closed using 1-0 and 4-0 absorbable sutures.

\section{CT measurement of cross-sectional area}

Seventeen participants ( 9 from the neurotization group, 8 from the non-neurotization group) in this study underwent 64-channel abdominal CT (Somatom Sensation, Siemens Medical Solutions, Erlangen, Germany) before surgery for preoperative planning and all 19 patients underwent postoperative CT. A single investigator measured a selected portion of the rectus muscle (at the second lumbar vertebra level) by means of ImageJ software in a series of 10 cross-sectional slices. The ImageJ program is a Java-based image analysis software package, which is freely downloadable from the US National Institutes of Health website (http://imagej.nih.gov/ij/). Then, the average of the measured areas was calculated (Figs. 3, 4).

The mean area of the rectus muscle was $406.69 \pm 67.01 \mathrm{~mm}^{2}$ on the right side and $400.98 \pm 74.02 \mathrm{~mm}^{2}$ on the left side on preoperative $\mathrm{CT}$ scan. No significant difference was observed in the mean area of the two sides $(\mathrm{P}=0.124)$ (Fig. 5). On the assumption that both sides of the rectus abdominis muscle were symmetric, the postoperative change in the rectus muscle was concluded to have occurred coincidentally. The contralateral rectus muscle was used as a control group in order to reflect the muscle change by various postoperative factors. For example, the muscle could have atrophied due to a shortage of exercise after the operation.

Attenuation of the skeletal muscle in vivo determined by CT 
scan is related to its lipid content. Therefore, this change may affect the value of the Hounsfield unit (HU), which we compared between the pre- and postoperative groups.

The areas of both sides of the rectus abdominis muscle were compared for investigation of their symmetry in the 17 patients who underwent CT scan before surgery. Differences between the remnant rectus abdominis muscle and contralateral rectus abdominis muscle as a control were obtained for evaluation of the effect of neurotization.

\section{Electromyographic analysis}

An EMG (Medelec Synergy, Viasys Healthcare Ltd., Warwick, UK) was performed for investigation of the contractile function of the remnant and contralateral rectus abdominis muscles. $\mathrm{Pa}$ tients were laid on the examination table in a supine position and the palpable site of the rectus abdominis muscle was examined. Then the patients underwent ultrasonography to determine the exact location of the remnant rectus muscle. Ultrasonographyguided needling was performed at exactly the site where the rectus abdominis muscle was observed. After checking that the

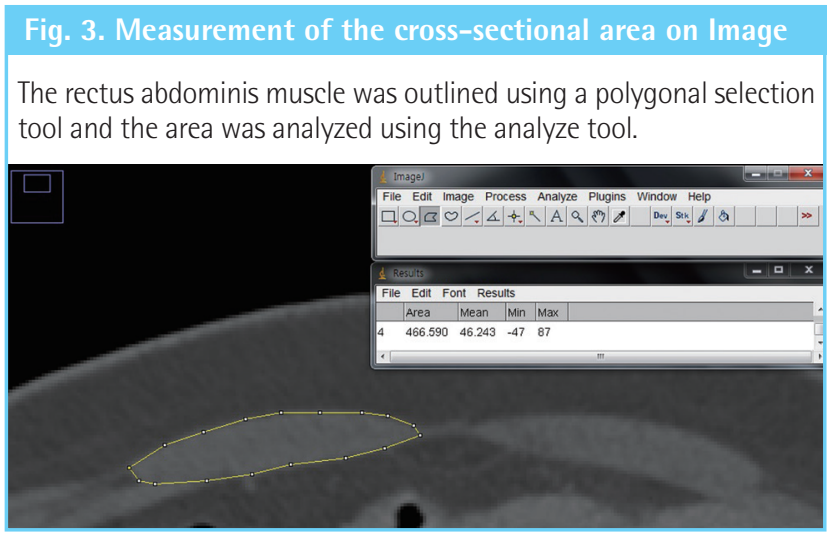

needle was placed in the rectus abdominis muscle, the examiner recorded the contractile waveform with the patients raising their head and leg. In the patients whose muscle could not be easily observed on ultrasonography, EMG was performed with placement of the needle as close as possible to the rectus fascia.

\section{Statistical analysis}

SPSS statistical software ver. 18 (SPSS Inc., Chicago, IL, USA) was used for analysis. The mean area and HU value of the rectus abdominis muscle were compared using Fisher's exact test and the Wilcoxon signed rank test. The proportion of patients with total atrophy of the rectus abdominis muscle was calculated and analyzed for statistical significance using Fisher's exact test. Probabilities of less than 0.05 were considered significant. Data were expressed as mean \pm standard deviation.

\section{Fig. 5. Symmetry of both sides of the rectus muscle}

Differences in the area of the rectus abdominis muscle between the right and left side were not statistically significant $(P=0.124)$.

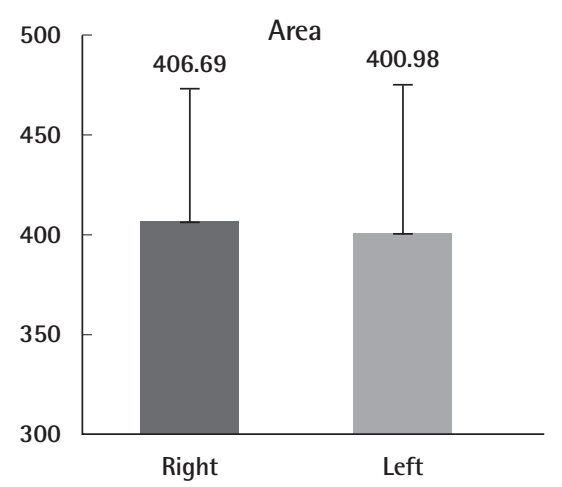

\section{Fig. 4. Postoperative CT of neurotization and non-neurotization groups}

(A) A 48-year-old patient of the neurotization group: a postoperative computed tomography (CT) scan showed a well-preserved ipsilateral rectus abdominis muscle (red arrow). (B) A 60-year-old patient of the non-neurotization group: a postoperative CT scan showed total loss of the ipsilateral rectus abdominis muscle (blue arrow).
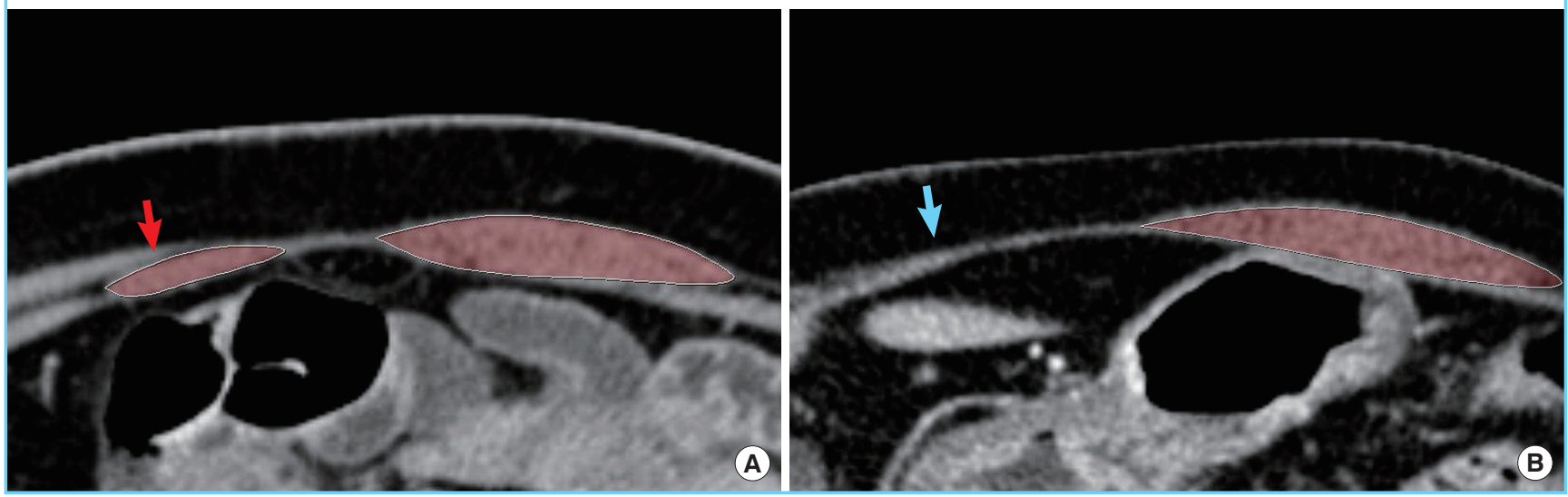


\section{RESULTS}

The mean age of the patients who underwent neurotized pedicled TRAM at the time of the examination was 48.4 years (range, 38 to 61 years) and that of the non-neurotized pedicled TRAM patients was 47.1 years (range, 37 to 60 years). The mean followup period was 21.7 months (range, 6 to 41 months) and 32.5 months (range, 20 to 52 months), respectively. No significant difference in age or follow-up period was observed between the groups $(\mathrm{P}=0.604$ and $\mathrm{P}=0.095$, respectively). The mean body mass index (BMI) was 23.29 and 22.96 in the neurotization and non-neurotization groups, respectively. No statistically significant differences were observed $(\mathrm{P}=0.780)$. There were no current or ex-smokers, and one patient in each group had a history of previous abdominal operation (Cesarean section) (Table 1).

\section{Cross-sectional area and $\mathrm{HU}$ value on postoperative CT} In 2 of the 9 patients in the neurotization group (22.22\%), the remnant rectus abdominis muscle totally atrophied and only the rectus sheath was observed. In the other patients (77.78\%), the average cross-sectional area of the remnant rectus muscle and contralateral muscle were $96.25 \mathrm{~mm}^{2}$ and $377.21 \mathrm{~mm}^{2}$, respectively. The difference in the mean area between the muscles was $281.73 \mathrm{~mm}^{2}$.

Atrophy of the remnant rectus abdominis muscle was observed in 8 of 10 patients ( $80 \%)$ in the non-neurotization group. In the other 2 patients, the cross-sectional areas of each of the 2 remnant rectus muscles were $72.16 \mathrm{~mm}^{2}$ and $90.46 \mathrm{~mm}^{2}$. The difference in the mean area could not be obtained due to a lack of subjects. However, statistical difference was observed in the proportion of total atrophy cases between the neurotization and non-neurotization groups $(\mathrm{P}=0.027)$ (Table 2$)$.

The authors originally planned to investigate the difference in the mean area between the neurotization and non-neurotization groups to compare the muscle preserving effect of neurotiza-

\section{Table 1. Summary of the patient characteristics}

\begin{tabular}{|lccc|}
\hline Characteristic & Neurotization & $\begin{array}{c}\text { Non- } \\
\text { neurotization }\end{array}$ & P-value $^{\text {a) }}$ \\
\hline No. of patients & 9 & 10 & - \\
Mean age (yr) & $48.4 \pm 8.41$ & $47.1 \pm 7.19$ & 0.604 \\
Follow-up length (mo) & $\begin{array}{c}21.67 \pm 13.57 \\
\text { (median 20) }\end{array}$ & $\begin{array}{c}32.50 \pm 12.29 \\
\text { (median 29) }\end{array}$ & 0.095 \\
Mean body mass index & $23.29 \pm 2.99$ & $22.96 \pm 2.90$ & 0.780 \\
Smoking history & 0 & 0 & - \\
Abdominal operation & 1 (C-sec) & 1 (C-sec) & - \\
\hline $\begin{array}{l}\text { No significant difference was observed in mean age, follow-up length, or mean } \\
\text { body mass index. } \\
\text { C-sec, Cesarean section. } \\
\text { a)Mann-Whiteny U test. }\end{array}$ & & \\
\hline
\end{tabular}

tion. However, the difference in the mean area after surgery was not comparable between the neurotization and non-neurotization groups because the authors could not obtain the mean value of the difference in the non-neurotization group given that only two cases did not completely atrophy.

In the 9 patients who had remnant rectus abdominis muscles, the values of $\mathrm{HU}$ were $45.14 \pm 6.18$ and $41.74 \pm 4.85$ in the remnant rectus abdominis muscle before and after operation, respectively. In the contralateral rectus abdominis muscle, the values were $46.78 \pm 3.77$ and $42.87 \pm 7.68$, respectively (Fig. 6). After surgery, there was a decrease in the value of $\mathrm{HU}$ in both groups and statistical significance was observed in the remnant rectus abdominis muscle $(\mathrm{P}=0.028)$. However, no significant difference was observed in the contralateral rectus abdominis muscle $(P=0.066)$. There was a mild decrease in the $\mathrm{HU}$ value of the neurotized remnant rectus muscle. However, the HU value of the neurotized remnant muscle was within the normal range.

\begin{tabular}{|c|c|c|c|c|c|c|}
\hline \multirow{2}{*}{ Patient ID. } & \multicolumn{3}{|c|}{$\begin{array}{c}\text { Neurotization } \\
\text { (postoperative) }\end{array}$} & \multicolumn{3}{|c|}{$\begin{array}{l}\text { Non-neurotization } \\
\text { (postoperative) }\end{array}$} \\
\hline & IRM & CRM & $\begin{array}{l}\text { CRM- } \\
\text { IRM }\end{array}$ & IRM & CRM & $\begin{array}{l}\text { CRM- } \\
\text { IRM }\end{array}$ \\
\hline 1 & 62.40 & 366.10 & 303.70 & 0.00 & 587.26 & - \\
\hline 2 & 82.41 & 338.36 & 255.95 & 0.00 & 310.00 & - \\
\hline 3 & 75.08 & 371.86 & 296.77 & 0.00 & 306.40 & - \\
\hline 4 & 180.58 & 470.73 & 290.15 & 72.16 & 422.47 & 350.32 \\
\hline 5 & 0.00 & 366.49 & - & 0.00 & 400.59 & - \\
\hline 6 & 40.79 & 317.82 & 277.03 & 0.00 & 363.44 & - \\
\hline 7 & 0.00 & 382.53 & - & 90.46 & 403.83 & 313.37 \\
\hline 8 & 102.40 & 414.71 & 312.30 & 0.00 & 511.16 & - \\
\hline 9 & 130.07 & 366.25 & 236.19 & 0.00 & 348.33 & - \\
\hline 10 & - & - & - & 0.00 & 409.64 & - \\
\hline Total atrophya) & $\begin{array}{c}2 / 9 \\
(22.22 \%)\end{array}$ & Average & 281.73 & $\begin{array}{l}8 / 10 \\
(80 \%)\end{array}$ & Average & $\begin{array}{c}\text { Not } \\
\text { available }\end{array}$ \\
\hline
\end{tabular}

\section{Fig. 6. Comparison of Hounsfield values}

After surgery, there was a decrease in the HU in both groups. A statistical difference between the preoperative and postoperative value was observed in the ipsilateral muscle $(P=0.028)$. $H U$, Hounsfield unit.

\section{Hounsfield units}

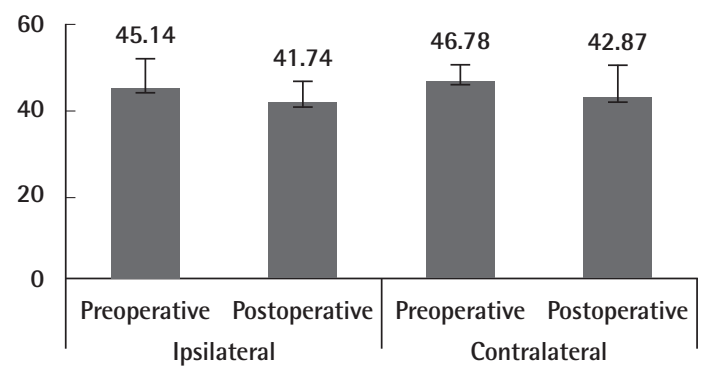




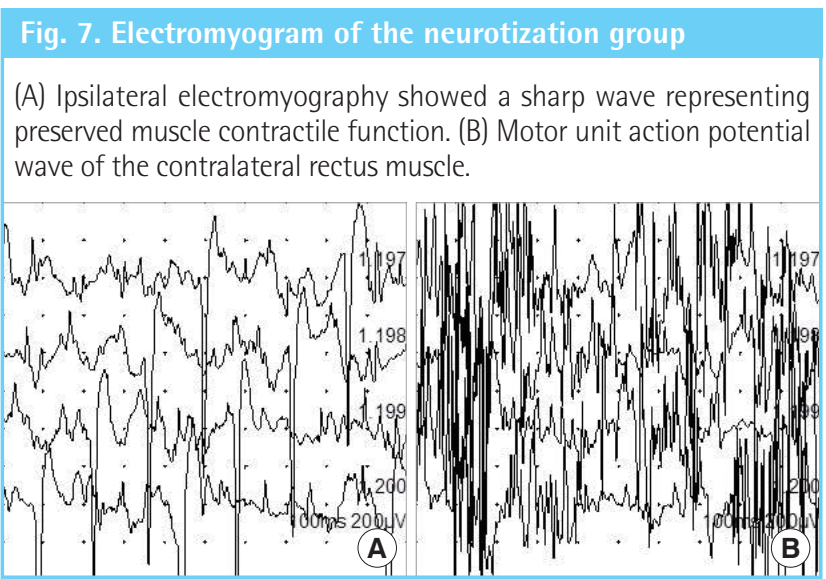

\section{Contractile function on EMG}

The motor unit action potential (MUAP), which measures the contractile function of muscle, was observed in all 9 patients with remnant rectus abdominis muscles as detailed in Figs. 7, 8.

\section{DISCUSSION}

Further reduction in donor site morbidity was introduced with the preservation of the lateral one third of the rectus abdominis muscle by Hartrampf [3]. Fewer complications of the donor site, compared with conventional pedicled TRAM, had been expected with this selective procedure. Duchateau et al. [12] reported that CT performed less than 6 months after operation showed the presence of remnant rectus abdominis muscle. However, after 6 months or more, CT failed to reveal any structure other than the fascia. The authors reported that the intercostal nerves, which were innervated at the medial aspect of the superior and inferior epigastric arteries, were injured. Therefore, the author reported that preserving the lateral strip of the rectus muscle was an unnecessary procedure.

On the other hand, Galli et al. [13] reported that penetration of the intercostal nerve to the rectus abdominis muscle had individual viability. Furthermore, in 19 of 31 patients, the lateral strip of the rectus abdominis muscle was observed six months postoperatively, even if the ninth intercostal nerve was injured during surgery [13]. The author reported that even though denervated muscle could not have contractile function, persistence of a muscular structure with its fascia could improve the abdominal wall competence from a static point of view. However, this article has limitations, such as the fact that the author considered only the ninth intercostal nerve, but, in reality, penetration of more than one intercostal nerve to the rectus muscle could be found during the operation.

Atrophy of the muscle was induced by denervation or ischemia. Insertion of intercostal nerves to the rectus abdominis muscle
Fig. 8. Electromyogram of the non-neurotization group

(A) Electromyography of the ipsilateral rectus abdominis muscle showed prolonged duration and low amplitude of motor unit action potential. Although the remaining muscles had contractile function, the prolonged duration and low amplitude indicated the possibility of reduced muscle fibers and nerve fibers. (B) Contralateral electromyography showed the sharp MUAP wave of intact muscle.

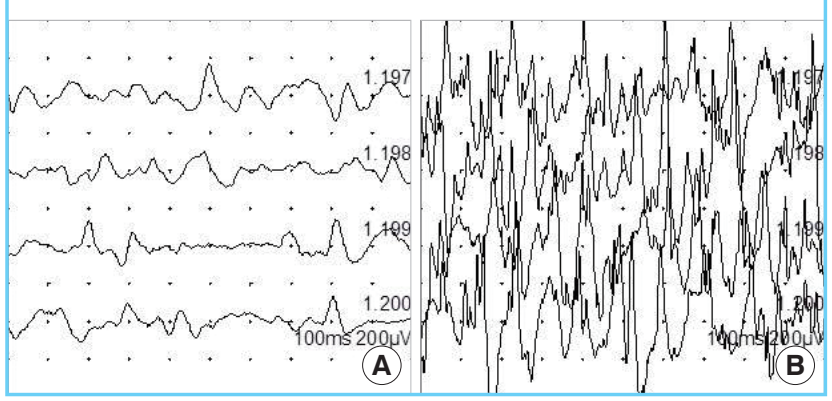

is correlated with the lateral row of the intramuscular pedicle passing along the longitudinal axis of the muscle. The lateral row of the deep inferior epigastric artery was located near the insertion of the intercostal nerve $[9,12,14]$. Therefore, the intercostal nerves were susceptible to damage when the medial two thirds of the rectus abdominis muscle was elevated. The vascularity of the remnant muscle was maintained from the deep fascia [12]. Indeed, a healthy muscle color and bleeding from the remnant muscle were observed after splitting of the rectus abdominis muscle. Therefore, it is reasonable that denervation of muscle can be considered to be a major cause of muscle atrophy.

We neurotized the intercostal nerve in order to preserve the lateral strip of the rectus abdominis muscle. Rozen et al. [15] reported two types of intercostal nerve. Small intercostal nerves can be sacrificed without functional deficit due to overlapping innervation from other intercostal nerves. However, a large one located around the arcuate line acts on the entire width of the rectus abdominis muscle without adjacent overlap. Therefore, we attempted to preserve this large intercostal nerve; if it could not be preserved because of insertion close to the pedicle, this nerve could be severed and transposed to the lateral third portion by neurotization.

The mean area of the rectus abdominis muscle was used to determine the degree of atrophy, and EMG of the remnant muscle was performed for assessment of contractile function. Before surgery, there was no significant difference in the mean area between the rectus abdominis muscle on the two sides (Fig. 5). Thus it makes sense to postulate that if both rectus muscles are symmetric, then the non-operated muscle can be used as a control in evaluating the change in the operated muscle. After the operation, the difference in the mean area between the two sides of the rectus abdominis muscle was obtained in the neurotization and non-neurotization groups. Only 2 remnant rectus abdomi- 
nis muscles were preserved during the follow-up period in the non-neurotization group. Therefore, the mean difference in the area between the two sides of the rectus abdominis muscle after the operation could not be calculated.

We expected that denervated rectus muscles would be observed to have fibrosis or fatty degeneration; however, the remnant rectus muscles had totally atrophied. Fleckenstein et al. [16] reported that denervation-related muscle change in magnetic resonance imaging (MRI) became more conspicuous with time in the early period of 1 to 12 months postoperatively and relatively little change occurred after 13 months. As previously stated, Duchateau et al. [12] found only fascial structure six months after muscle sparing pedicled TRAM. The minimum follow-up period was six months and all but 3 patients had over 12 months of follow-up in this study. Therefore, there was sufficient time for monitoring the denervation effect on the muscle.

We can determine the content of structures such as muscle and bone from the $\mathrm{HU}$ value in CT. Attenuation values are expressed in $\mathrm{HU}$ and ranged from 31 to $54 \mathrm{HU}$, depending on the lipid content [17]. Denervation of the rectus abdominis muscle is associated with fatty degeneration [16]. After operation, the HU value of the remnant rectus abdominis muscle had slightly decreased with statistical significance, from $45.14 \pm 6.18$ to $41.74 \pm$ 4.85. The remnant rectus abdominis muscle had more lipid content after surgery, but the $\mathrm{HU}$ value was also within the normal range of muscle. Although there was a decrease in the HU value, it did not necessarily mean fatty degeneration of the muscle because the $\mathrm{HU}$ value remained in the normal range for muscle after the operation. However, even though there was no statistical significance, the $\mathrm{HU}$ value of the contralateral muscle had also decreased postoperatively, from $46.78 \pm 3.77$ to $42.87 \pm 7.68$. We can assume that the accumulation of intramuscular lipids increased because the usage of the abdominal wall declined for many possible reasons such as abdominal weakness, physical condition, or lifestyle change. However, our study was not designed to test for the factors influencing the $\mathrm{HU}$ change.

The lateral strip of the rectus abdominis muscle was preserved in two patients in the non-neurotization group after operation. The follow-up periods were 39 and 52 months, respectively. Although there was sufficient time for development of atrophy of the rectus abdominis muscles, their muscle bulk remained. The intraoperative photograph shows how the 2 rectus muscles could have been preserved by preservation of the intercostal nerves, including the large intercostal nerve at the arcuate line (Fig. 2A, B). In the author's experience, however, when the rectus muscles were harvested safely, intercostal nerves were more than likely damaged.

EMG shows the duration and amplitude of MUAP. The dura- tion of MUAP better reflects the number of muscle fibers and the amplitude reflects the distance between the needle and the muscle fiber. Therefore, these values vary according to the location of the needle, and the amount and diameter of the muscle fiber [18]. For this reason, observation of MUAP on EMG indicates that the muscle has contractile function; however, quantitative analysis of the contractile function cannot be performed. In EMG of 9 remnant rectus abdominis muscles that were preserved after surgery, 4 remnant rectus abdominis muscles generated MUAP waveforms similar to the contralateral rectus abdominis muscle, and the others showed a prolonged duration and low amplitude compared with the contralateral rectus abdominis muscle (Figs. 6, 7). Because the EMG could not measure the strength of muscle quantitatively, this result does not necessarily indicate weakness of contractile function. However, further investigation to examine the relationship between the EMG waveform and neurotization is needed because both of the two remnant rectus abdominis muscles that were preserved after surgery in the non-neurotization group showed a prolonged duration and low amplitude of MUAP. From this result, we can recognize that there is contractile function of the remaining remnant rectus abdominis muscle and the absence of denervation-related atrophy.

In pedicled TRAM, it may be helpful to maintain a portion of the rectus muscle for abdominal wall competence. Mizgala et al. [5] reported that $46 \%$ of their patients felt a loss of abdominal strength after undergoing a pedicled TRAM operation. In contrast, Suominen et al. [7] reported that there was no difference in the abdominal wall strength between pedicled and free TRAM. Although previous studies have reported conflicting results, if the remnant rectus abdominis muscle has contractile function, it is reasonable that the remnant rectus abdominis muscle contributes to the strengthening of abdominal wall competence. Partial harvest of the rectus abdominis muscle that needs to fold at the pivot point of the flap ensures less epigastric bulging due to less muscle bulk and improves flap vascularity by less compression of venous drainage at the folded area of the rectus muscle [11]. In addition, the remnant rectus abdominis muscle behind the anterior fascia may facilitate closure with less fascial tearing by providing suture-holding tissue.

The lateral strip of the rectus abdominis muscle can be easily divided after identification of an intramuscular course of superior and inferior epigastric arteries. The neurotization was performed so that the severed nerve was buried in the muscle and closed with fine sutures. This procedure could be easily performed without microscopic assistance. We concluded that neurotization can be performed without additional time and effort. However, there were drawbacks to this study. First, a small 
number of subjects were included in this study. Second, further evaluation is needed in order to investigate the correlation between the remnant rectus abdominis muscle and abdominal wall competence.

Neurotization to reduce the degree of atrophy was found to reduce the number of patients with atrophy. Neurotization also contributed to preserving the normal quality of muscle after surgery. In conclusion, we suggest that neurotization is an appropriate procedure when it is performed together with the muscle-sparing pedicled TRAM flap.

\section{REFERENCES}

1. Hartrampf CR, Scheflan M, Black PW. Breast reconstruction with a transverse abdominal island flap. Plast Reconstr Surg 1982;69:216-25.

2. Hartrampf CR Jr, Bennett GK. Autogenous tissue reconstruction in the mastectomy patient: a critical review of 300 patients. Ann Surg 1987;205:508-19.

3. Hartrampf CR Jr. Abdominal wall competence in transverse abdominal island flap operations. Ann Plast Surg 1984;12: 139-46.

4. Grotting JC, Urist MM, Maddox WA, et al. Conventional TRAM flap versus free microsurgical TRAM flap for immediate breast reconstruction. Plast Reconstr Surg 1989;83: 828-41.

5. Mizgala CL, Hartrampf CR Jr, Bennett GK. Assessment of the abdominal wall after pedicled TRAM flap surgery: 5- to 7-year follow-up of 150 consecutive patients. Plast Reconstr Surg 1994;93:988-1002.

6. Serletti JM, Moran SL. Free versus the pedicled TRAM flap: a cost comparison and outcome analysis. Plast Reconstr Surg 1997;100:1418-24.

7. Suominen S, Asko-Seljavaara S, von Smitten K, et al. Sequelae in the abdominal wall after pedicled or free TRAM flap surgery. Ann Plast Surg 1996;36:629-36.

8. Alderman AK, Kuzon WM Jr, Wilkins EG. A two-year prospective analysis of trunk function in TRAM breast reconstructions. Plast Reconstr Surg 2006;117:2131-8.

9. Hammond DC, Larson DL, Severinac RN, et al. Rectus abdominis muscle innervation: implications for TRAM flap elevation. Plast Reconstr Surg 1995;96:105-10.

10. Lejour M, Dome M. Abdominal wall function after rectus abdominis transfer. Plast Reconstr Surg 1991;87:1054-68.

11. Clugston PA, Gingrass MK, Azurin D, et al. Ipsilateral pedicled TRAM flaps: the safer alternative? Plast Reconstr Surg 2000;105:77-82.

12. Duchateau J, Declety A, Lejour M. Innervation of the rectus abdominis muscle: implications for rectus flaps. Plast Reconstr Surg 1988;82:223-8.

13. Galli A, Adami M, Berrino P, et al. Long-term evaluation of the abdominal wall competence after total and selective harvesting of the rectus abdominis muscle. Ann Plast Surg 1992; 28:409-13.

14. Moon HK, Taylor GI. The vascular anatomy of rectus abdominis musculocutaneous flaps based on the deep superior epigastric system. Plast Reconstr Surg 1988;82:815-32.

15. Rozen WM, Ashton MW, Kiil BJ, et al. Avoiding denervation of rectus abdominis in DIEP flap harvest II: an intraoperative assessment of the nerves to rectus. Plast Reconstr Surg 2008; 122:1321-5.

16. Fleckenstein JL, Watumull D, Conner KE, et al. Denervated human skeletal muscle: MR imaging evaluation. Radiology 1993;187:213-8.

17. Goodpaster BH, Kelley DE, Thaete FL, et al. Skeletal muscle attenuation determined by computed tomography is associated with skeletal muscle lipid content. J Appl Physiol 2000;89:104-10.

18. Dumitru D, Amato AA, Zwarts MJ. Electrodiagnostic medicine. 2nd ed. Philadelphia: Hanley \& Belfus; 2002. 